\title{
Teoría de la Argumentación como Epistemología Aplicada
}

\author{
ARgumentATION THEORY AS Applied EPISTEMOLOgY \\ Dr. Cristián Santibáñez (cristian.santibanez@udp.cl) Centro de Estudios de la Argumentación y el \\ Razonamiento, Facultad de Psicología, Universidad Diego Portales (Santiago, Chile)
}

\begin{abstract}
In this paper the conception of argumentation theory as applied epistemology is discussed. The point of departure is the description of four perspectives that are considered as founders of the modern theory of argumentation, in order to observe whether there was a similar concept in those theories or if they provided the patterns to go into that direction. Further on the reasons why contemporary scholars have given this emphasis to the notion of argumentation theory is discussed.
\end{abstract}

Key words: argumentation, dialectics, epistemology, informal logic, normativity

\section{Resumen}

En este artículo se discute la visión de la teoría de la argumentación como una forma de epistemología aplicada. El punto de partida es la descripción de cuatro perspectivas que se consideran fundadoras de la teoría moderna de la argumentación, para desde allí observar si en ellas hubo un concepto similar o si se dieron las pautas para tomar esa dirección. Luego, se reflexiona en torno a las razones por las que los teóricos posteriores han dado énfasis a esta visión de la teoría de la argumentación.

Palabras clave: argumentación, dialéctica, epistemología, lógica informal, normatividad

\section{Introducción}

La teoría de la argumentación ha experimentado un crecimiento y desarrollo exponencial. Bien se tematice en filosofía como problema en la lógica, se incorpore en la sociología como dispositivo metodológico (como un tipo de análisis del discurso), se plantee como área de investigación en psicología del razonamiento, o se enseñe en lingüística como expresión de la corriente pragmática. Esto ha ocurrido a pesar de que no existe una historiografía exacta del concepto teoría de la argumentación. Precisamente, quizás sea este el indicio que ha permitido a parte del mainstream en ciencias sociales y las humanidades tomar una posición escéptica o de cautela frente a un escenario académico de vertiginoso ascenso que, benignamente, se presenta como respuesta disciplinar o experta a problemas de corte científico y social, como se verá luego (1).

Si bien la tradición en retórica y dialéctica y las explicaciones lógicas en el siglo XX del lenguaje natural en términos de demostración y estructura silogística no cuentan como referencia directa a la noción de teoría de la argumentación, obviamente forman parte del sustrato básico respecto del que ésta obtiene su robustez.

De acuerdo con algunas panorámicas del estado del arte en este campo de estudios (Blair 2011, Cantú y Testa 2006, van Eemeren, Garssen, van Haften y Krabbe 2012, Johnson 2000, Rehg 2009, Walton, Reed y Macagno 2008, Walton 2007), donde se atestigua que convergen las dimensiones tradicionales 
mencionadas con otros desarrollos más novatos (psicología del razonamiento, filosofía del lenguaje, pragmática, teoría de la comunicación), se aprecia que el concepto de teoría de la argumentación no sólo tiene un nacimiento difuso, sino además un uso reciente, probablemente a partir de algunos esfuerzos reflexivos germanos y holandeses que, hacia fines de 1970, utilizaron el concepto de forma explícita y constante (Berk 1979, Kopperschmidt 1980, Öhlschläger 1979, van Eemeren, Grootendorst y Kruiger 1978, Schecker 1977). Sin embargo, un rastreo bibliográfico más acucioso daría con la publicación del retórico y filósofo americano Johnstone (1968), quien utiliza la noción teoría de la argumentación para referirse, precisamente, a la revalorización del trabajo filosófico atendiendo a criterios retóricos y argumentativos.

Ciertamente, la cronología bibliográfica es secundaria a la hora de entender el auge disciplinario de la teoría de la argumentación, sobre todo cuando hubo movimientos estructurales en la academia americana y europea que iban de la mano de demandas sociales en pos de cambios en los currículos universitarios. En Canadá, en la década de 1970, el movimiento de la lógica informal ya había dado frutos sustanciales en el estudio del razonamiento, las falacias y el argumento como un tipo producto mental y social (Johnson y Blair, Woods y Walton), en función de las modificaciones que se pedían en los departamentos de sociología, psicología y filosofía a fines de 1960. Hacia mediado de la década de 1980, se utilizaba en habla inglesa, casi sin distingo, las nociones de lógica informal, teoría de la argumentación y/o teoría del argumento (Govier, Walton) para referirse al conjunto de iniciativas reflexivas en torno al razonamiento práctico y cotidiano, que fue lo pedido por la juventud intelectual de las décadas de 1960 y 1970 en gran parte del mundo occidental.

En Estados Unidos hubo una historia similar a la de Canadá: los movimientos sociales en California dieron un impulso incontrarrestable a iniciativas académicas que, hasta entonces, eran minoría (como los trabajos de Rescher y Wenzel), que buscaban derrocar la primacía del estudio de la lógica formal como parámetro para el entendimiento de la validez filosófica y comunicativa. Pero la reflexión alrededor de la teoría de la argumentación en ese país estuvo siempre apoyada por una tradición única en retórica que, probablemente vinculada a un contexto sociocultural de deliberación sistemática, permitió un despegue institucional de mayor envergadura que perdura hasta hoy en los departamentos de comunicación, periodismo y retórica, en los que la teoría de la argumentación es parte obligada en las licenciaturas. No es casualidad, entonces, que el primer ensayo o libro publicado bajo este nombre, teoría de la argumentación, se haya dado aquí con el trabajo de Willard.

En Francia, la propuesta de Anscombre y Ducrot, sin indicación absoluta a los colegas canadienses o americanos ya mencionados, se orientó al estudio de la argumentación desde una perspectiva lingüística que, en el inicio, tampoco usó la nomenclatura teoría de la argumentación para referirse a lo que hacían. Dentro de Europa, será en los Países Bajos donde la teoría de la argumentación conozca el programa más ambicioso e interesante, cuyos creadores, quizás hacia 1996 (van Eemeren y Grootendorst), no dejaron dudas respecto de que la teoría pragma-dialéctica era una teoría de la argumentación y no sólo una aproximación al discurso argumentativo. Este programa no ha parado de crecer y aun no se vislumbra un debilitamiento importante en su quehacer. Pero como siempre es el caso, la escuela pragma-dialéctica tiene deudas teóricas innegables que se podrían resumir en los siguientes nombres: Hamblin, respecto del problema de las falacias; Barth y Krabbe, respecto de la dimensión normativa y la lógica del diálogo; y Searle, respecto del acento pragmático en el uso del lenguaje.

Más allá de la comunidad específica de cultores que se dedica a la teoría de la argumentación, hay cuatro nombres que aun no se mencionan pero que son citados en muchas esferas y discusiones de las disciplinas de las ciencias sociales y las humanidades una vez se entra a los fundamentos de este campo: Arne Naess, Rupert Crawshay-Williams, Chaim Perelman y Stephen Toulmin. En efecto, siendo más o menos explícito el reconocimiento, en cualquier propuesta dentro de lo que contemporáneamente se llama teoría de la argumentación estas referencias son ineludibles, y este trabajo no será la excepción. 
Pero en el contexto de esta amplia comunidad de procedencia, y en virtud de los acentos disímiles entre las aproximaciones, aun persisten preguntas básicas, a saber: ¿qué es la teoría de la argumentación?, ¿una construcción dentro de los campos de estudio de la comunicación?, ¿un cuerpo de ideas que proviene de la preocupación por el discurso?, ¿una explicación del razonamiento en términos sociales?, ¿una explicación dialógica del funcionamiento de las controversias?, ¿un nuevo nombre para una antigua preocupación en la retórica?, ¿la versión lingüística y filosófica del trabajo en la argumentación jurídica?, ¿la versión lingüística y filosófica de la teoría de la decisión y la psicología del razonamiento?, o ¿una propuesta epistemológica respecto de la revisión y justificación de creencias?

Porque no es posible, ni deseable, responder a estas preguntas con un: es todas a la vez y ninguna en específico, lo que aquí se presenta tratará en particular el caso de la teoría de la argumentación como expresión de una epistemología aplicada. Para el efecto, en la siguiente sección se describe el estado general de la comunidad científica que cobija a los estudios de la argumentación para observar su grado de madurez; en el tercer apartado, se exponen y discuten los conceptos básicos de los cuatro teóricos seminales arriba señalados, con el objeto de preparar la discusión específica y observar qué hereda a las aproximaciones de última generación; y en la cuarta sección, se reflexiona específicamente en torno a la visión y definición de la teoría de la argumentación como epistemología aplicada. En la sección conclusiones, se retoman los puntos esenciales y se proyectan nuevas preguntas con el objetivo de discutir y abrir otras líneas de investigación y análisis, en particular el problema socio-epistémico de la teoría de la argumentación.

\section{El escenario de los estudios de la argumentación}

No es aventurado señalar que en el campo de los estudios contemporáneos de la argumentación en el siglo $\mathrm{XX}$, tres de las cuatro perspectivas (Naess, Toulmin y Perelman) que inauguraron la reflexión reaccionaron, de un modo u otro, a las crisis provocadas por las dos guerras mundiales, por el ascenso político de regímenes totalitarios en el Este Europeo y por el nacimiento del uso masivo de la propaganda política y comercial y las consecuencias de estos hechos en el trabajo y respuestas científicas a los problemas contingentes (el estudio de la propaganda política en el siglo XX ha recibido una atención especial entre los analistas del discurso, quienes han utilizado encuadres teóricos provenientes de modelos de análisis de la argumentación, como también retóricos y pragmáticos). Como se planteaba en la introducción, la teoría de la argumentación se ha presentado como un refuerzo académico a la práctica deliberativa para conseguir una democracia sana (como es el convencimiento de Johnson). No es un misterio, en la ardua discusión en la arena sociológica, que el intento de Habermas por hacernos entender el funcionamiento de la pragmática universal iba en consonancia con la necesidad de una ética discursiva, sobre la base de reglas de argumentación, para la pertinencia comunicativa y el logro de la comprensión intersubjetiva. Nótese que Habermas cita a Toulmin en esto.

En 1941 el filósofo y lógico noruego Arne Naess publica en su lengua materna Comunicación y argumento. Elementos de semántica aplicada, que en 1966 se traduce al inglés, sentando ciertas bases para una teoría dialógica de los juegos conversacionales, lo que será retomado por los formalistas del diálogo y los pragmadialécticos. Pero, ¿qué contiene este trabajo que lo hace pública o socialmente relevante? ¿A qué demanda responde? Desde un punto de vista social, sin lugar a dudas, la importancia de su trabajo radica en que formula las primeras reglas para a una discusión pública y crítica en contextos de uso del lenguaje natural, adelantándose con mucho las propuestas de última generación. Un dato no menor respecto de la obra de Naess es que pasa de las preocupaciones por la lógica al ecologismo y se convierte en el fundador temprano del movimiento "ecología profunda", lo que no es una casualidad ni un capricho personal: Naess fue de aquellos filósofos y ciudadanos que hacían de su pensar y obrar una pareja coherente con la demanda contextual.

Quizás menos huellas de consonancia entre su vida intelectual y el estado epocal se puede observar en la biografía del británico Rupert Crawshay-Williams quien, sin embargo, publica dos monografías que develan 
claramente una intención acorde con una inconformidad académica: The Comforts of Unreason (1947) y Methods and Criteria of Reasoning. An Inquiry into the Structure of Controversy (1957), siendo este último el antecedente a los intentos por identificar la formas de uso del lenguaje en tanto instrumento de la razón para la definición de temas y apreciaciones científicas.

Por su parte, Chaim Perelman y Lucien Olbrechts-Tyteca, en Bélgica, y Stephen Toulmin en Inglaterra, en 1958, dieron impulso a sus propuestas pensando siempre en describir y criticar el modo en que la tarea científica se llevaba a cabo, desenmascarando los fantasmas e ilusiones que crearon tanto el acento exacerbado en el razonamiento de la lógica matemática en la reflexión científica, como el desconocimiento de las formas específicas que adquieren los argumentos en la ciencia y las estrategias retóricas que se usan en su afán. Mientras Perelman y Olbrechts-Tyteca remodelaron el aporte de la retórica sistematizando figuras clásicas en el marco de nuevas categorías y conceptos para indicar la diferencia fundamental existente entre demostración y argumentación, Toulmin optó, en el marco de un ensayo sobre el status epistemológico de las ciencias de la conducta, por el desmembramiento de la estructura del argumento mostrando cuán cerca está el razonamiento de las ciencias de una lógica jurídica generalizada antes que de una lógica matemática. Hasta donde se sabe, el azar condujo a que las obras principales de estos autores se publicaran en el mismo año: 1958. Los títulos originales son: La Nouvelle Rhétorique: Traité de l'Argumentation para el texto de Perelman y Olbrechts-Tyteca, y The uses of argument para el texto de Toulmin. Ninguno se planteó en términos de una "teoría de la argumentación".

De allí en más, como toda la crítica especializada lo acentúa, los desarrollos en Europa y Estados Unidos florecieron. Asociados estos desarrollos a ángulos diversos, según se fueron recapitulando folios de la retórica, la dialéctica y sumando los de la pragmática -e incluso algunos de la semiótica-, la teoría de la argumentación fue cobrando reputación, por una parte, por su marcado interés reflexivo, pues se presentó como un campo académico específico -a pesar de que confluyeron muchas áreas disciplinarias-, y por otra parte, por su irrenunciable compromiso práctico de aplicación, pues se erigió como cuerpo teórico con metodologías al servicio de problemas reales en el análisis de textos, discursos y comunicaciones en general, en particular al servicio del análisis y evaluación del debate racional.

Se debe hacer notar que para muchos la argumentación como campo de estudio también debe su renovación a una serie de esfuerzos de organización disciplinaria y curricular. En 1979 la Speech Communication Association de Estados Unidos organizó una conferencia sobre argumentación en Utah, siendo sus actas una muestra general sobre el estado de la cuestión hacia la época; sin embargo, en 1986, y bajo el alero de la Internacional Society for the Study of Argumentation (ISSA), se celebró la primera conferencia de argumentación con sede en la Universidad de Ámsterdam, Países Bajos, la que cada cuatro años reúne a los más prestigiosos estudiosos del área en un mismo lugar. No obstante, desde un punto de vista estrictamente académico, es la Ontario Society for the Study of Argumentation (OSSA), con sede en la Universidad de Windsor, Canadá, la que desde 1995, y cada dos años, realiza la conferencia de mayor impacto intelectual en el área, reuniendo a los especialistas para que expongan sus avances en temas específicos dado un tópico de convocatoria (2).

Actualmente, los grupos de investigación de mayor influencia (como la escuela de Lugano, Ámsterdam, Dundee, Windsor, Chicago, entre otras), están orientando tesis doctorales y proyectos de estudio a áreas específicas, con estrecho vínculo a problemas sociales, tales como la aplicación de técnicas argumentativas al servicio de la comunicación en salud, en el sistema financiero, en las aplicaciones tecnológicas, en las reformas a los sistemas jurídicos, en los currículos educativos y en la reflexión política en torno a la democracia deliberativa. Como se observa, los esfuerzos en este campo han ido desembarcando en las arenas de discusión social más sensibles de una época en la que la duda y la acción ciudadana han renovado sus fuerzas. 


\section{Desde los primeros bosquejos contemporáneos}

Como se observa, la diseminación de la teoría de la argumentación sigue los tres caminos usuales: extensiones teóricas vinculando reflexiones y dominios científicos (psicología, sociología, género, lingüística, inteligencia artificial, etc.), contrastaciones empíricas (para observar, por ejemplo, si los hablantes de una lengua natural usan o no determinados esquemas argumentativos, acusan recibo y reprueban movimientos falaces, utilizan y perciben sesgos argumentativos, etc.), y aplicaciones e intervenciones en la sociedad (tanto tecnológicas -a través de software educativos-, como en virtud de la creación de protocolos para mediaciones legales, o metodologías de intervención médica).

Todo este cuadro promisorio tuvo un comienzo, o al menos un renacer temático, de corta data. No es necesario retrotraerse a los griegos o medievales, sino por el contrario la escena contemporánea es, hasta cierto punto, autosuficiente en sus aperturas y derroteros para haber creado este despegue. $Y$ en efecto, a partir de las cuatro propuestas que a continuación se pasan a resumir, varias de las extensiones mencionadas se podrían explicar en sus puntos cardinales.

\subsection{La semántica aplicada de Arne Naess}

Naess puede ser clasificado como un filósofo experimental, corriente que hoy ha tenido un vigoroso revival y apoyo de los departamentos de sociología y psicología experimental. En esta forma de ver las cosas, sus investigadores consideran que los cuestionarios y las entrevistas -las metodologías de las ciencias sociales en general- son la mejor manera de adquirir información para entender cuál es el sentido de una expresión en un contexto determinado y cuáles, finalmente, son los problemas del razonamiento importantes de afrontar. En su inicial empirismo semántico, Naess promovió tres conceptos básicos para una teoría de la argumentación, los que luego por ejemplo fueron efectivamente integrados por la teoría pragma-dialéctica. Estos conceptos e ideas fueron: 1) Antes que un desacuerdo pueda ser resuelto, las incomprensiones entre los que discuten deben ser eliminadas; para este efecto, Naess (1966) propone una serie de reglas de procedimiento dialéctico; 2) Para lograr la clarificación, se requiere llevar a cabo un proceso de "precisación" (neologismo que viene a traducir precization del inglés), lo cual quiere decir, un acto de interpretación en el que se asigna una proposición a una formulación, proposición que debe estar en acuerdo con (ser aceptable para) los involucrados y las circunstancias (precisación es así una jugada comparativa en el marco de un diálogo); y 3) Para resolver una diferencia de opinión, los que discuten deben expresar tanto evidencia a favor como evidencia en contra del punto de vista que defienden para, de este modo, dar cuenta del peso de su posición. En esto último, Naess sigue la dialéctica clásica.

Para Naess, la calidad de la evidencia en un proceso argumentativo, que pueda realmente resolver una diferencia de opinión, depende de su fuerza justificativa y de su defendibilidad (tenability), y la defendibilidad de una evidencia depende, a su vez, de su valor de verdad, corrección y plausibilidad. Según Naess (1966:108ss.), la fuerza justificativa de una evidencia se mide por su potencial de prueba, es decir, por su relevancia.

Una de los problemas básicos que ataca Naess, es la de diferenciar cuidadosamente entre lograr acuerdo y tener un real de desacuerdo. Simplemente, en el mundo real, nos recuerda Naess (1966:83ss.), tendemos a quedarnos satisfechos por alcanzar pseudo-acuerdos (o pseudo-desacuerdos), que se originan por no verificar el sentido y alcance de las expresiones, por no revisar todas las consecuencias de una expresión, por no balancear (escuchar) todas las posiciones que puedan importar en una disputa, por un uso sostenido e invariante de eulogismos y/o dislogismos (esto es, expresiones con fuerte énfasis emocional positivo o negativo respectivamente).

De este modo, Naess ve la necesidad de promover cierta noción de código de conducta, tanto cognitivo como ético, para llevar a buen término un intercambio argumentativo. Desde el punto de vista cognitivo, habría para él dos formas de procesamiento argumentativo: pro-et-contra (a favor y en contra), y pro-aut- 
contra (a favor o en contra). En el primero, el argumentador debiera listar los argumentos a favor y en contra de una conclusión; en este contexto, recalca Naess, el hablante, en contextos naturales, no llega a una conclusión realmente, sino que se advierte, a través de un ejercicio como si fuera un observador externo, de las posibilidades disponibles para un flujo argumentativo. En el segundo, en cambio, el hablante sintetiza los argumentos más importantes para aducir en un momento dado, utilizando uno o varios de ellos a favor o en contra de una tesis específica. Usualmente, es lo que hacemos en contextos reales. Este ejercicio, en efecto, termina por plantear una conclusión. Ambas formas deben suponer, no obstante, el uso preciso de las formulaciones. Con esta distinción tan básica, Naess lo que único que hace es ofrecer una técnica elemental para hacerse cargo del razonamiento teórico, por una parte, y el razonamiento práctico, por otro.

Respecto de un código de conducta ética, Naess (1966:121ss.) lo presenta como principios para una discusión efectiva. Para el intercambio de contenidos cognitivos cuya meta es la convicción, estos principios o estándares son bases mínimas mas no exhaustivas: 1) Evitar las referencias tendenciosas hacia un solo lado atribuyéndole contenidos específicos; 2) Evitar la interpretación tendenciosa respecto de los contenidos de la otra parte; 3) Evitar la ambigüedad; 4) Evitar obtener implicaciones tendenciosas de un argumento; 5) Evitar un uso tendencioso de nuestros propios reportes o de la información de primera mano (no abusar del testimonio ni del argumento por autoridad); y 6) Evitar un uso tendencioso del contexto. Como se observa, con esto Naess incluso se estaba adelantando a las reflexiones en torno al sesgo cognitivo estudiado en psicología social y del razonamiento que desde los años de 1970 hacia adelante tuvo un boom a través de la noción de heurístico.

Por supuesto, no toda la propuesta de Naess es consistente y valiosa para el desarrollo de una teoría de la argumentación. Por ejemplo, hay una confusión en el autor respecto del uso de la idea de lo relevante. En relación con estos seis principios de corte ético, le llama a la conducta respetuosa de ellos relevante, y antes ya había utilizado la noción vinculándola con la fuerza justificativa de una evidencia. Del mismo modo, los principios son de tal grado generalistas que difícilmente se observe una práctica argumentativa no tendenciosa (éste es justamente el punto de los estudiosos de la heurística y la racionalidad limitada. Con todo, y obviamente, su marcado interés por la definición contextual de los términos para una discusión, la necesidad de principios guías que orienten el actuar argumentativo, la urgencia por develar pseudoacuerdos, y también la urgencia por estudiar el problema semántico de la comunicación bajo la cautela de la responsabilidad intelectual abierta a la práctica real, hacen de este temprano esfuerzo una piedra angular en la teoría de la argumentación contemporánea. De hecho, la pragma-dialéctica es, en cierto sentido, su extensión y debe bastante a su memoria.

\subsection{Controversia y necesidad metodológica en Rupert Crawshay-Williams}

¿Por qué ciertos tipos de controversias teóricas y sociales son tan odiosamente intratables? ¿Por qué algunos desacuerdos son difícilmente resueltos? Dos preguntas que cualquier investigador social se hace continuamente y que Crawshay-Williams hacia la década de 1950 comenzaba a responder. Por cierto, su contribución no dio totalmente con una respuesta satisfactoria, toda vez que las preguntas aun persisten cultural y técnicamente, pero avanzó en dos interesantes direcciones.

Primero, sostuvo que la falta de resolución es producto de la inexistencia de criterios a partir de los que un juicio puede ser testeado. Y porque no hay entre los hablantes un criterio base, las incomprensiones se profundizan. Segundo, a nivel técnico, sostuvo que una categorización preliminar de criterios puede pensarse tridimensionalmente a través de: un criterio lógico, un criterio convencional y otro empírico.

El primero, se refiere a aquellas reglas del razonamiento aceptadas, tácita o explícitamente, por las partes y que son pensadas como orientadoras de inferencias válidas (por ejemplo, que las partes consideren que los razonamientos que apelan a la ignorancia son inválidos, o aquellos en los que se afirma el consecuente sean débiles). Por su parte, usar un criterio convencional es utilizar una proposición o juicio respecto del que la 
otra parte está en acuerdo de forma previa, y este acuerdo en juicios compartidos se puede referir a definiciones aceptadas por ambos, a procedimientos ya establecidos o por efecto de una negociación. Por último, el criterio empírico se puede entender cuando se divide en dos dimensiones: el criterio objetivo, esto es, que las proposiciones deben concordar con los hechos, y el criterio contextual, esto es, que la forma en que los hechos se han descrito debe estar en concordancia con el propósito de la proposición esgrimida (Crawshay-Williams 1957:34-36). Para despejar cualquier duda de un objetivismo ingenuo en CrawshayWilliams, una diferencia nunca puede resolverse sólo observando la correspondencia entre el status de verdad de una proposición y los hechos que dice ocupar, sino que siempre es necesario observar el propósito para los que tales hechos son descritos, en otras palabras, el criterio contextual debe ser aplicado.

La otra dirección de trabajo abierta por Crawshay-Williams parte del dato real que, en situaciones cotidianas, las discusiones tienen, y abundantemente, proposiciones indeterminadas. Esto conlleva obviamente el peligro del mal entendimiento entre los hablantes, porque desde el momento en que los contextos de las expresiones se mantienen muchas veces implícitos o no expresados (que es también el problema de la vaguedad), las interpretaciones y abusos interpretativos se generan rápidamente. Para salvar este impase, el autor propone que los participantes de una discusión deben asumir los diferentes contextos de una proposición. Aunque parezca una banalidad, en términos de tiempo real, lo que rara vez pasa es que los participantes en efecto dejen por explícito qué contexto para la proposición tienen en mente. Evidentemente, nosotros tendemos a igualar nuestro contexto de la proposición con el contexto universal o aquel fuera de duda.

Tanto desde un punto de vista lógico, convencional o empírico, muchas reglas específicas de estos criterios tienen una necesidad metodológica. Por necesidad metodológica, el autor se refiere a la existencia de niveles de ordenamiento y jerarquización. Esto quiere decir lo siguiente: si la lógica nos enseña que "una proposición no puede ser al mismo tiempo falsa y verdadera" (principio de no contradicción), esto es sólo así si las partes han aceptado, tácita o explícitamente, este principio y han hecho uso de él.

De modo que uno de los grandes corolarios de la postura del británico es que la validez de varios principios y criterios en el mundo real, depende de consideraciones metodológicas que los participantes de una discusión negocian o aceptan tácitamente al ser parte de una comunidad de habla. En otras palabras, Crawshay-Williams aporta al debate de la racionalidad dentro de la teoría de la argumentación dos ideas fundacionales: 1. La racionalidad argumentativa para hablantes naturales debe tener una base convencional intersubjetiva, y 2 . Debe tener también una base contextual objetiva.

\subsection{Audiencia y persuasión en Perelman}

El hacer intelectual de Perelman fue prolífero, erudito, relevante y comprometido políticamente. Así lo atestigua su recepción en la jurisprudencia, en la teoría de la comunicación, en la semiótica, en la teoría política, en la reflexión histórica y por supuesto en la teoría de la argumentación. De inmediato, se debe dejar constancia que en una de sus principales contribuciones a la teoría de la argumentación, La Nouvelle Rhétorique: Traité de l'Argumentation (2000), escrita conjuntamente con Lucien Olbrechts-Tyteca, los autores citan a Arne Naess y Rupert Crawshay-Williams como antecedentes.

Precisamente, como lo indica el título de la obra, su trabajo se conoce bajo el nombre neorretórica, porque es la versión contemporánea de los esfuerzos griegos y latinos. La línea inaugurada por Perelman y Olbrechts-Tyteca se caracterizó por su fuerte acento en la idea de que el estudio de la argumentación está destinado a distinguir el set de técnicas dispuestas en un discurso por un hablante para obtener la adhesión de la audiencia. Como atestiguan las referencias bibliográficas del libro, la obra fue la consecuencia natural de una larga trayectoria académica que, al parecer, comenzó cuando el autor investigaba la relación entre derecho, retórica y lógica, derivando desde allí a los problemas de la comunicación, el discurso y la argumentación. Destacaremos un aspecto del extenso catastro expuesto por estos autores belgas. 
En la tercera parte del libro, que versa sobre las técnicas de la argumentación, se exponen cinco apartados consagrados a los mecanismos más importantes que Perelman y Olbrechts-Tyteca lograron sistematizar (2000:187ss). Reflexionar en torno a las técnicas de la argumentación es preocuparse por las articulaciones reales que construyen los hablantes para hacer de sus discursos algo efectivo. Algunas de ellas funcionan de forma inconsciente, como parte de una competencia general, y otras son utilizadas de forma deliberada, calculando los espacios y las maniobras posibles. Mencionar y explicar brevemente las técnicas estructurales que a continuación se exponen, sólo busca mostrar el abanico de distinciones que Perelman y OlbrechtsTyteca llegaron a concebir:

1) los argumentos cuasi-lógicos: en esta categoría se explican los problemas de la contradicción e incompatibilidad de los argumentos; los temas de la identidad, definición, analiticidad y tautología de los argumentos; las nociones de transitividad, inclusión y división de los argumentos; y el problema de la regla de justicia y reciprocidad;

2) los argumentos basados en la estructura de lo real: distinción que se fundamenta en los modos de enlace de los argumentos y donde se presentan las nociones de: enlaces por sucesión, por coexistencia, doble jerarquía y diferencias de orden en los enlaces;

3) los argumentos que fundamentan la estructura de lo real: esto es, aquellos argumentos que, a partir de un caso, permiten generalizaciones, siendo los conceptos claves: ejemplo, ilustración, modelo, analogía y metáfora;

4) la disociación de las nociones: que se concibe como una técnica de transformación de incompatibilidades de los conceptos involucrados en los argumentos;

5) la noción de interacción de argumentos: que hace alusión a la conexión e interacción constante entre los argumentos (en términos de cantidad, orden y adecuación) tanto en los que constituyen la audiencia como en el espacio mental del hablante que intenta mostrase coherente y así lograr la adhesión.

En ciencia hay muchos ejemplos para graficar cada una de estas técnicas. Particularmente, los argumentos que fundamentan la estructura de lo real son de uso frecuente: las metáforas son propicias para explicar fenómenos físicos y químicos (3); en filosofía existe una tendencia similar al hablar, a través de analogías, de juegos de lenguaje (4). Otro uso extensivo, por ejemplo en la discusión pública (política o cultural), es lo que pasa cuando un orador coordina su discurso bajo la estrategia de la definición, esto es, usa un argumento a partir del que se avanza una elección particular del sentido y alcance de un problema en una proposición.

Para mostrar el camino que sigue la acción y el conocimiento en la ciencia, Perelman y Olbrechts-Tyteca insisten en la peculiaridad de que toda empresa de convicción supone el contacto intelectual. Este lugar común se debe repetir: se argumenta para un auditorio específico, y en ese afán, la distinción de los marcos que una comunidad comparte, la asimilación de las bases de acuerdo del contexto, son procedimientos básicos para tener éxito. Sin embargo, la tendencia a sobreestimar este aspecto de la actividad argumentativa -a saber la dependencia con el contexto y la audiencia-, nos hace olvidar la reflexión de Perelman y Olbrechts-Tyteca en torno a la categoría auditorio universal, cuyo sentido se vincula con la idea de que un discurso racional o razonable apela, inevitablemente, a ser entendido y aceptado por todo aquel que posee juicio, capaz de aceptar razones y evidencia. Como se observa, esta idea, finalmente, está vinculada con un estándar epistémico que tiene dos entradas: aquel que produce discurso -en el caso de la ciencia, el que busca producir conocimiento- tendrá que entregar buena evidencia a su favor; aquel que recibe un argumento con pretensiones universales de validez debe estar inclinado a aceptarlo cuando así la fuerza de las buenas razones lo obliguen. Buena evidencia a su favor tiene aquí una denotación flexible. Incluso podría vincularse con la noción de un código de conducta argumentativa, tal como lo propuso Naess. Un código de conducta argumentativa quiere decir que, en virtud de cierta discusión -en particular en la ciencia-, el hablante sólo debería utilizar algunas estructuras argumentativas, en particular aquellas que 
eviten falacias -tales como los argumentos por autoridad, o aquellos que apelan al gusto popular; del mismo modo, el hablante debería esforzarse por exponer ciertos esquemas argumentativos -un argumento causal no es tan simple de insertar en explicaciones en las ciencias sociales.

El control del auditorio es un problema crucial en Perelman. Enfatiza no olvidar que un hablante no necesariamente, y bajo toda circunstancia, se dirige a un auditorio presente o explícito, pues puede ser parte de su intención y estrategia dirigirse a un auditorio ausente o futuro (lo hacen los políticos por ejemplo). Lo importante para Perelman siempre fue debilitar la posición filosófica y social que asumió un racionalismo cartesiano sin matices, ya que a su juicio en esa tradición hubo una desestimación de la posibilidad de deliberar a partir de argumentos plausibles. Considerar como primordial la categoría de verdad como criterio para juzgar y evaluar argumentos, nunca fue uno de sus consejos.

\subsection{Toulmin: El argumento es como un organismo}

Escribió Toulmin en 1958: An argument is like an organism. Como ya lo advirtiera Perelman y OlbrechtsTyteca a propósito del uso de técnicas que fundamentan la estructura de lo real (en este caso de las ciencias y las humanidades), con esta metáfora Toulmin no sólo ilustraba analógicamente su concepto de argumentación, sino que develaba su visión general del lenguaje, en una obra que ha sido considerada fundacional en el campo de los estudios de la argumentación: The Uses of Argument.

Su visión general estaba fuertemente marcada por un acento pragmático, tanto en el sentido de lo práctico como de lo útil. Esta visión consideraba esencial preguntarse por las condiciones reales y vitales en las que cada empresa humana comienza y, en particular, construye sus coordenadas de validez. Fue producto fidedigno, sino impulsor, del giro lingüístico y tomó seriamente el desafío de investigar la idea de que el significado del lenguaje es su uso, pregonado por uno de sus profesores: Ludwig Wittgenstein.

La consistencia metafórica de lo que implicaba esta oración, la cuidó finamente Toulmin a lo largo de este texto y en otras de sus obras, ya que concibió que los argumentos tienen partes (como la forma o el esqueleto de un organismo), que los argumentos se mueven por campos argumentativos -como los de la ciencia, la economía o el arte-, que los argumentos se reproducen con mayor facilidad en ciertos contextos -como en las conferencias científicas o las reuniones de negocios o los tribunales-, que los argumentos tienen enfermedades (las falacias), que los argumentos tienen cierta longevidad según estén abiertos a la crítica -que les da mayor expectativa de vida y que Toulmin distinguió como argumentos sustanciales-, o ciegos y sordos a la crítica -que los hace diluirse con mayor rapidez porque no tienen cabida en muchos contextos y que Toulmin consignó como argumentos analíticos. Todo este encuadre metafórico, y lo que tiene de particular The uses of Argument, se orienta hacia la tarea de develar cómo las ciencias de la conducta (sociología, antropología, psicología, etc.) estructuran su conocimiento, justifican sus pretensiones y respaldan sus juicios.

Convengamos de inmediato que lo que se encuentra en su texto es un modelo que distingue las partes de un argumento para el entendimiento del procedimiento racional en ciencias. Sin embargo, frente a la recepción, especialmente la academia norteamericana de los departamentos de retórica y comunicación, Toulmin et al publica en 1979 un libro en el que intenta señalar qué aspectos debiera cubrir una teoría de la argumentación. En este segundo texto, los autores definen argumentación y argumento, hablan de falacias, de esquemas argumentativos, aplican el modelo en diferentes contextos y casos, profundizan en los conceptos de campo invariable y campo variable, de fuerza y criterio, y distinguen distintos ámbitos de razonamientos -el legal, el científico, el ético, el económico, y el artístico. De allí en más, se ha tomado el modelo original y los conceptos de sus publicaciones posteriores como un conjunto teórico específico. El concepto clave para entender la posición general de Toulmin sigue siendo, no obstante, el de lógica factual. Por tal término cabría entender que la argumentación es un procedimiento sustancial, nunca formal, regido por los estándares fácticos que se despliegan en cada contexto de proposiciones y racionalidad (en estudios con perfiles más históricos y sociológicos, Toulmin reemplaza el concepto de 'racionalidad' por 
'razonabilidad' para enfatizar la dimensión práctica del razonamiento verdaderamente importante). El modelo sirve, entonces, para desnudar el apoyo sustancial de cada argumento, su procedencia, su fuerza y criterio de aplicación. Cada categoría del modelo -a saber, dato, cualificadores modales, condiciones de refutación, conclusión, garantía y respaldo-, cumple un objetivo puntual en el desmantelamiento de los argumentos, mostrando un engranaje que poco tiene que ver con la lógica silogística y mucho con el razonamiento habitual de la jurisprudencia (los alcances más importantes del modelo y perspectiva de Toulmin han sido analizados en un volumen editado por Hitchcock y Verheij, en el que investigadores de distintas áreas discutieron críticamente aspectos epistemológicos, retóricos y lógicos de la propuesta toulminiana).

La propuesta de Toulmin es una discusión epistemológica de la empresa científica no sólo porque el autor lo declara explícitamente (1958:211-252), sino porque, y esencialmente, intenta mostrar cómo se justifica una creencia. Más específicamente, para Toulmin las situaciones epistemológicas dan lugar a preguntas de diferente índole. Una cosa es avanzar una pretensión de saber, otra es crear relaciones de justificación entre razones y la pretensión de saber, otra cosa es observar si la justificación dada responde a una norma, si merece ser aceptada o si debe ser rechazada. Intuitivamente, los hablantes cuando avanzan una proposición, con la pretensión de saber que es verdad, implican que las razones que podría exponer en apoyo son de relevancia y tienen fuerza. Muchas veces la fuerza de las razones son independientes del campo bajo el que cabe una pretensión, porque se puede vincular con la forma de la aseveración, pero la relevancia es dependiente del contexto, ya que su adecuación es cuestión de criterio de aplicación.

Uno de los grandes problemas con lo que se enfrenta la teoría epistemológica en general, es lo que Toulmin llama tipo lógico. Por ejemplo, hacemos aseveraciones sobre el futuro y las apoyamos con datos del presente y del pasado; hacemos aseveraciones sobre la naturaleza y las apoyamos con los resultados de observaciones y experimentos particulares; es decir, mientras presentamos las proposiciones que damos por conocidas a partir de un tipo lógico, los apoyos y datos provienen de otro. ¿Cómo se resuelve este problema? Toulmin no da una respuesta acabada del asunto del que se ocupa (ya le había pasado a Naess y Crawshay-Williams en sus respectivos temas), pero nos llama a no confundir los contextos en que se ponen en cuestión estos vínculos aparentemente inadecuados (como por ejemplo respaldar aseveraciones obre el futuro con datos del pasado). Toulmin lo grafica y explica con el siguiente ejemplo: "Un hombre mira hacia adelante desde su automóvil y ve que la carretera está despejada, entonces le hace una señal al coche de detrás para que lo adelante. Ver la carretera despejada le proporciona una razón para actuar así: lo primero es la justificación de lo segundo. Pero aunque ver consista en una cosa y hacer señales en otra, no hay "laguna" entre el acto de ver y el de hacer señales: sólo hay una diferencia. Para justificar nuestra señal sólo tenemos que fijarnos en el estado de la carretera: no tenemos también que proporcionar principios ulteriores para salvar el vacío entre la visión y el acto. La pregunta práctica ahora no es: ¿Puede el acto de señalizar llegar a ser por sí mismo equivalente a ver, o el de ver al de señalizar?, sino ¿En qué casos el hecho de ver algo justifica una actividad (enteramente distinta) de señalización?" (1958: 251).

\section{La argumentación como epistemología aplicada}

La pregunta práctica de Toulmin es la pregunta que la epistemología aplicada intenta responder. ¿Qué es la epistemología aplicada? De acuerdo con Fumerton (2006), es aquella que trabaja sobre el problema de qué es lo que sabemos y cómo la sabemos (qué cosas intervienen en el acto de saber), mientras que, en contraposición, la meta-epistemología se pregunta qué es el conocimiento mismo, reflexiona metateóricamente respecto de qué puede llegar a nombrase como conocimiento y qué significa, para un agente, estar en conocimiento de algo: qué es tener una certeza de conocer algo y cómo se concibe una creencia.

Las analogías, nuevamente, ayudan a entender lo implicado -a costas de cierta simplificación: mientras le ética se pregunta por las distinciones entre buen y mal comportamiento, la meta-ética por las propiedades intrínsecas o naturales del comportamiento bueno y malo, en el caso de que existan tales propiedades. La epistemología aplicada, de este modo, se pregunta por: ¿sabemos y/o conocemos, o creemos 
justificadamente que lo hacemos $-\mathrm{y}$ si es así cómo- proposiciones sobre el pasado, el futuro, el mundo externo, las otras mentes? Muchas veces, las fronteras se cruzan para beneficio de ambos lados: la epistemología aplicada tiene en su seno discusiones de orden meta-reflexivo y la preocupación metareflexiva utiliza proposiciones de primer orden para lograr consistencia teórica.

En el caso de la teoría de la argumentación esto se traduce a un dominio en el que se pesquisa cómo una creencia está justificada y a leer el output de la argumentación como el resultado de dar (buenas) razones para aceptar y creer una pretensión. Es crucial entender que la práctica argumentativa misma está epistemológicamente orientada cuando vincula la pretensión de conocimiento tanto con creencia justificada como con condiciones de verdad. Dicho en simple: un hablante no sólo dice que sabe algo porque tiene una buena razón para justificar lo que sabe, sino además porque considera implícita o explícitamente que esa creencia cumple con ciertas condiciones de verdad, condiciones que pueden estar contenidas en el tipo de acto de habla a través del que la proposición de saber tuvo expresión (generalmente, a través de una aserción). Obviamente, un hablante puede decir que sabe algo sabiendo al mismo tiempo que es mentira, pero este mismo hecho ya le hace sospechar que su argumentación es mala, débil o de fácil crítica. Sólo se auto-engañan epistémicamente aquellos agentes están sometidos a constricciones severas de algún tipo (sicológicas y/o sociales).

Desde este punto de vista, no es difícil ver el legado de las cuatro piedras angulares de la teoría de la argumentación comentadas en la sección anterior. Cada autor presupuso aportar a la noción de buena argumentación, analizando estrategias dialécticas y retóricas para lograrlo, definiendo marcos metodológicos para resolver ciertos puzles, observando y tratando de aclarar problemas básicos en la ecología argumentativa, insistiendo en la necesidad de contextualizar la práctica argumental y el análisis conceptual.

Tampoco es casualidad que algunos investigadores actuales del área (Battersby), retomando este legado, conciban que la tarea de la teoría de la argumentación es saber guiarse por la práctica argumentativa contextualizada, pues allí se encontrarían los desafíos teóricos y las normas específicas de una buena argumentación. Por supuesto, en la literatura contemporánea, no hay acuerdo en absoluto respecto de qué caracteriza una buena argumentación (Govier, Lumer). Hamblin (1970: 224-252), por ejemplo, consideró que una buena argumentación debería cumplir con tres criterios: 1) criterio alético: que las razones del argumento sean verdad, y que las razones lógicamente impliquen la conclusión; 2) criterio epistémico: el oyente sabe que las razones son -o pueden ser- verdad y que la tesis se sigue claramente de tales razones; y 3) el criterio retórico: el oyente acepta las razones. Estrictamente hablando, ninguno de estos criterios es satisfactorio desde un punto de vista epistemológico. El problema fundamental es que no se introduce en estos criterios el razonamiento plausible, aquel cuyas premisas no son del todo conocidas como verdaderas ni tampoco se sabe si pueden llegar a serlo pero que, no obstante, arroja conocimiento fiable sobre la base de un respecto a toda la información básica de la base de datos desde donde proviene el argumento (Lumer, 2005: 194). Base de datos aquí significa contexto.

Dentro de las visiones de la teoría de la argumentación como un tipo de hacer epistemológico aplicado, las diferencias también se observan (Battersby, Biro y Siegel, Goldman, Freeman, Johnson, Weinstein). Donde sí existe acuerdo es en la necesidad de incluir una sub-teoría general de la falacia dentro de cualquier marco teórico de la argumentación.

Una teoría de la falacia es la contrapartida negativa a la idea de buena argumentación. Ciertamente, sobre la definición de falacia hay también matices. Una posición agnóstica de la falacia, sostendría que es un procedimiento general usado para fijar creencias que tiene una tendencia alta e inaceptable de generar creencias falsas o infundadas; o una posición funcional de la falacia la definiría como aquel acto de habla que obstaculiza la resolución crítica de una diferencia de opinión (van Eemeren y Grootendorst), o como el descarrilamiento de una maniobra estratégica (van Eemeren); una definición más general y común de falacia la concibe como un argumento que parece válido pero no lo es (Hamblin). Cualquiera sea la definición, lo 
importante a enfatizar es que el uso de la noción de falacia, así como la mera idea de una buena argumentación en el seno de la teoría de la argumentación, es indicativa de su orientación normativa sine qua non.

La teoría de la argumentación es el caso de un tipo de epistemología aplicada porque distingue los criterios normativos desde y para la práctica argumentativa, discusiva y contextualmente situada. Sus distinciones provienen tanto de la caracterización en abstracto del funcionamiento del lenguaje y de categorías analíticas (como por ejemplo, la descripción de los actos de habla), como del levantamiento y análisis de la validez intersubjetiva, es decir, de los patrones que hablantes naturales disciernen como apropiados o inapropiados en un intercambio y que, de acuerdo con algunas investigaciones (van Eemeren, Garssen y Meuffels), coinciden entre diversas ecologías argumentativas. Incluso si observamos nuestro propia actividad argumentativa, entonces daríamos con evidencia a favor de esta posición y, además, con algunas de las funciones estándares de avanzar un argumento e involucrarse en una argumentación: 1) llevar al oyente hacia una creencia racional o razonablemente justificada (como se cree ahora mismo se trata de hacer), esto es, guiar al oyente en el proceso de aceptación o que, en su defecto, se de cuanta de la verdad o aceptabilidad de la tesis (la "pretensión" en la nomenclatura de Toulmin); y 2) desarrollar, así se necesite o se nos pida en un intercambio, las razones ulteriores por las que creemos que algo es un buen argumento, es decir, explicitar los criterios que distinguen, para cierto contexto y meta, un argumento efectivo.

De lo anterior se debe esclarecer un elemento teórico sensible y que muchas veces tiende a confundir, ya que la noción de argumento podría verse teñida por una ambivalencia y hacer pensar, de esta forma, que es sinónima tanto de la noción de creencia como de la noción de argumentación. Existe un status cognitivo de tener una creencia, que tradicionalmente se conoce en filosofía de la mente como un tipo de estado intencional, y el proceso mental que la acoge en un sujeto podría compartir con la noción de argumento el que manifiesta una representación (a veces incluso la disposición inicial hacia un curso de acción), pero una vez que se gatilla algún proceso de revisión de tal creencia, impulsado frecuentemente por una irritación externa, alguna información del ambiente o enviada por otros agentes, se pasa corroborar o cuestionar su status de verdad y se observa si cumple con ciertos criterios mínimos de aceptabilidad para una audiencia u oyente. En ese preciso instante, el estado intencional creencia como representación cognitiva deja de ser el mismo el estado intencional representado. Dicho de forma más directa: si el estado intencional creencia es una tesis con orientación argumentativa, el proceso de justificarla la convierte en argumento y su dimensión social -para una audiencia- la convierte en argumentación. De modo que el proceso frugal de hacer funcionar creencias para tomar decisiones automáticas e intuitivas no es, obviamente, el objeto natural y básico de la teoría de la argumentación.

La dirección retórica de Perelman respecto de lo que hace de un argumento algo bueno, la aceptación por parte de la audiencia, tenía en potencia un criterio epistémico. Y cuando Toulmin et al (1979:13) definieron argumento como la cadena de razonamientos o secuencias interconectadas entre pretensiones y razones que establece el contenido y fuerza de la posición a partir de la que un hablante arguye, y argumentación como la actividad total de exponer pretensiones, desafiarlas, apoyarlas produciendo razones y nuevamente criticar esas razones, no hacían otra cosa que describir un fenómeno distinto respecto del estado intencional creencia. El proceso de someter a prueba socialmente nuestras tesis entre agentes razonables es el único que asegura un cambio en el contenido y fuerza de la posición de un agente, es muy inusual que, incluso en el más inteligente de los agentes, se produzca algún cambio en su aislamiento cognitivo. No es casualidad que en la psicología del razonamiento se haya llegado a esta misma conclusión después de muchos años de resultados experimentales.

Cuando los autores seminales de la teoría de la argumentación describían y explicaban la actividad de evaluar puntos de vista entre hablantes naturales como expresión de una interacción dialógica, no sólo incluían una intuición sociológica, a saber su condición social, sino también una psicológica, en el sentido de que asumieron que la actividad argumentativa está marcada tanto por el carácter crítico-cognitivo del 
agente involucrado en el proceso, como por la condición de auto-convicción del agente de pensar que su punto de vista es mejor (más fuerte, más aceptable, más razonable, más relevante) que el de su oponente, puesto que de otra forma un agente no entra a participar en tal tipo de actividad comunicativa. Es precisamente esta doble condición, social y psicológica, la que entrega la normatividad basal que caracteriza la argumentación.

Varios autores contemporáneos en la teoría de la argumentación ven en esto último el punto central de la teorización general, como de la visión del campo en tanto epistemología aplicada: el problema de la función, o funcionalidad, de la argumentación. Uno de los reconocimientos más claro de esta tendencia es la concepción que hay sobre la lógica informal y la argumentación en los desarrollos de la Escuela de Windsor (Johnson, Johnson y Blair, Walton). Ciertamente, hay matices entre los investigadores que constituyen este grupo, siendo el caso que algunos dudan si en efecto la teoría de la argumentación puede ser vista solamente como epistemología (Johnson). Con todo, la definición de la lógica informal y argumentación en esta posición manifiesta un funcionalismo característico. Por lógica informal se ha entendido un brazo de la lógica cuya función, tarea y objetivo es desarrollar estándares no-formales, criterios y procedimientos para el análisis, interpretación, evaluación, crítica y construcción de la argumentación en el discurso cotidiano. Uno de los elementos teóricos medulares de este acercamiento es que el modelo informal se alza como una alternativa a la noción de validez deductiva al poner el acento en tres criterios para evaluar la relación entre premisas, conclusión y audiencia: 1) relevancia, 2) suficiencia, y 3) aceptabilidad. El primer criterio dice relación con el examen de la existencia o no de una relación sustantiva, pertinente, entre premisas y conclusión; el segundo, apunta a la verificación de si acaso las premisas proveen de suficiente evidencia para la consecución de la conclusión; y el tercero, se vincula con el examen de si las premisas son, en tanto tales, verdaderas, probables o engañosas y si fluyen en la circunstancia en la que han sido apeladas. Incluso más contundentemente en su apreciación respecto de los beneficios funcionales de argumentar, Johnson sostiene que aquellos que participan en una argumentación incrementan su poder racional, desarrollan un sentido de respeto por la evidencia, desarrollan también un sentido de respeto por las diferencias de opinión y un aprecio por la conducta crítica. Goodwin, por el contrario, cuestiona sin vacilación que la argumentación, y el argumento en particular, tenga los beneficios y efectos que se asumen. Goodwin, discutiendo la posición pragmática y normativa de Walton, duda si el contexto de un argumento deba ser concebido como una joint activity (actividad conjunta), que tal actividad conjunta tenga la función de alcanzar un valor social, y que la exposición de argumentos siga un conducto normativo respecto del que los hablantes estén, consciente o inconscientemente, siguiendo. Patterson confronta a su vez las ideas de Goodwin señalando que el funcionalismo en la teoría de la argumentación, como en varios campos científicos, solo puede ser falseado una vez que se tenga una teoría específica respecto de la que los datos reales vengan a cuestionar o desmentir.

Desde la psicología cognitiva también ha habido perspectivas al respecto. Recientemente algunos autores (Sperber, Mercier), han puesto en duda todos los avances provenientes de la teoría de la argumentación entre sociólogos, lingüistas y filósofos, al sostener que la argumentación y la inteligencia corren por módulos paralelos y no siempre funcionan de forma coordinada, por lo que los resultados de un proceso argumentativo no están determinados por una evaluación estrictamente crítica de la mano de una depuración racional; del mismo modo, sostienen que los humanos argumentamos siempre con un sesgo de confirmación y que la evidencia en psicología cognitiva de los últimos cuarenta años muestra que, debido a este sesgo, los humanos argumentamos aquello que podemos justificar y no lo que sabemos sería una mejor opinión o argumento. De acuerdo con estos autores, la argumentación no brinda ninguno de los beneficios y funciones positivas que a menudo se piensa tiene, y no cabría teorizarla como expresión o manifestación epistémica, pues no se rige por ningún estándar normativo virtuoso. Una de las conclusiones que más llama la atención de la posición de Sperber y su equipo, es que los humanos son en principio crédulos de aquellos en los que confían, pero tan luego como desconfían lo suficiente, recién allí resuelven cotejar más atentamente el contenido de los argumentos poniendo en práctica un escaneo de la coherencia y relevancia de lo comunicado. 


\section{Conclusiones}

En el escenario optimista, que la argumentación es una actividad cuya naturaleza muestra una normatividad orientada hacia la bondad, o en el escenario pesimista, que la argumentación es materia de sesgos, queda la impresión, totalmente justificada, que la idea de la teoría de la argumentación como epistemología aplicada es, finalmente, deriva de un acercamiento social. Dicho de otra forma, el acercamiento apropiado a la argumentación, desde un punto de vista epistemológico, sería lo colectivo. De hecho, hay quienes sostienen que esta posición sería la única que da buenos réditos para la reflexión epistemológica general (Gilbert, Taylor), como para la teoría de la argumentación en particular (Goldman).

En esta sección final se volverá a las preguntas planteadas en la introducción, así como a las respuestas de los teóricos fundacionales y contemporáneos, a partir de un contrapunto con la perspectiva de la epistemología social para observar un paralelo que abre nuevas líneas de investigación.

Por epistemología social cabría entender (Goldman) el estudio de las propiedades epistémicas de los individuos que aparecen cuando ellos se relacionan con otros. Las raíces de esta rama de estudio en filosofía y sociología tienen una notable dispersión. Sin embargo, cabe mencionar que en Charles Taylor y Margaret Gilbert se encuentran algunos de las premisas fundamentales (no obstante, estos autores titularon su acercamiento como "epistemología colectiva"). El caudal central de la reflexión epistemológica ha considerado que los agentes epistémicos son exclusivamente individuales, que los conceptos normativos clave son justificación, racionalidad y conocimiento, que los estándares normativos tienen una validez objetiva, que los conceptos normativos nombrados dan como resultado la verdad, que la verdad es un estado de cosas independiente del agente, que lo medular de la preocupación es la dimensión doxática de la toma de decisiones. Frente a esto, los epistemólogos sociales han tomado tres caminos de crítica: revisionismo, preservacionismo y expansionismo, respectivamente.

A través de la dirección revisionista, sus cultores consideran que el conocimiento es materia de conversación constante, por lo que mal cabe la posibilidad de encuentro con la verdad, que las creencias son asunto de saberes institucionalizados, histórica y culturalmente localizados, contexto en el que sólo cabe como práctica la consulta a parámetros relativos a las fronteras de las declaraciones del discurso bajo análisis. En esta postura, relativismo y contextualismo encuentran terreno fértil. Contribuciones de la sociología de la ciencia, el posmodernismo, el análisis del discurso, entre otros dominios, han armado esta forma de entender la construcción del conocimiento. ¿No es acaso la teoría de la argumentación una sub-propuesta de estos intentos epistemológicos toda vez que se consideran las prácticas colectivas como formatos básicos? Si la respuesta es positiva, la teoría de la argumentación sería una teorización producto de un movimiento más amplio de las ciencias sociales, y su crecimiento se debería a su fuerte énfasis operacional, metodológicamente hablando, ya que facilitaría el desmontaje de una actividad que por naturaleza el social: argumentar. La neo-retórica de Perelman tiene claramente atisbos de esto, y Toulmin a veces se considera tributario de un cierto contextualismo. Si la respuesta es negativa, entonces habría que construir una teoría de la argumentación que deje bien en claro que aspectos de la actividad de argumentar dependen de lo mental, y cuáles dependen de lo social.

En el preservacionismo, como su nombre lo indica, se trata de preservar algunas nociones de la epistemología tradicional, en particular la idea de que el proceso de formación y revisión de creencias es algo individual. Lo que se agrega es la evidencia social con la que los individuos desarrollan tales procesos. Dos fuentes de evidencia colectiva parecen ser principales en este acto: el testimonio y el desacuerdo por pares. A través del testimonio, un agente epistémico no sólo forma una creencia a partir de una fuente indirecta respecto del contenido específico de su proposición, sino que además va fortaleciendo un procedimiento de justificación: la confiabilidad del reporte de tercera mano. El desacuerdo con pares sitúa el problema de la revisión de creencias a luz de agentes que utilizan la misma evidencia para contradecirnos y tienen, a su vez, las mismas habilidades cognitivas que nosotros. De este modo, el preservacionismo 
considera que el factor social tiene un rol medular tanto en el juego de la justificación como en la racionalidad en la que se obtienen creencias. Algo de esta preocupación tuvo Crawshay-Williams.

En el expansionismo epistémico, los teóricos están preocupados por resolver qué cuenta como nueva evidencia en un proceso comunicativo, y cómo cabe introducirla en un diálogo. La teoría de la argumentación podría ser vista como una forma de tratamiento, precisamente, de este problema epistemológico. La propuesta de Walton y Krabbe respecto de los tipos de diálogos, los compromisos dialógicos involucrados y los conceptos básicos del razonamiento en interacción que subyacen en ellos, puede ser catalogada como una modelización que da cuenta del fenómeno. Nótese que en esto, los autores citan a Naess y apoyan sustancialmente la visión pragma-dialéctica de la argumentación que se ha esforzado por crear una teoría crítica de la resolución de diferencias de opinión en la que prime el orden comunicativo. La dimensión social de esta noción -el expansionismo- está dada en virtud de la siguiente explicación: porque los agentes buscan incrementar la utilidad -subjetiva- esperada en cualquier proceso de toma decisión, entonces los agentes evidentemente introducen nueva información, de acuerdo con ciertos grados de confiabilidad y razonabilidad, para obtener la decisión más consistente según cierto entorno. Confianza y razonabilidad aquí se refieren a la estimación de las fuentes de información, a las habilidades cognitivas de los involucrados y a los costos sociales que significa no introducir, en efecto, nueva información (evitar ser acusados de "negligencia epistémica").

Lo que viene a enfatizar la epistemología social, desde el punto de vista de la comunicación, es que la justificación de creencias razonables es un problema de justificación interpersonal, es decir, un problema dialéctico. El postulado reza como sigue: $\mathrm{S}$ está personalmente justificado en creer $\mathrm{P}$ si y sólo si tiene, o podría tener, éxito en justificar interpersonalmente $P$ frente aquellos que la desafíen, real o potencialmente. Este axioma, que sin contexto pareciera ser ingenuo, basa su fuerza también en un principio comunicativo implícito: en la confianza que quien desafíe una proposición cambiará su opinión si el agente $\mathrm{S}$ le da buenas razones para que lo haga. Puede ser el caso que un agente de buenas razones pero el oyente, por motivos psicológicos o institucionales de diferente orden, se niegue a aceptar la buena evidencia y/o justificación. Cualquiera sea el subsecuente proceso destinado a hacer cambiar de opinión al testarudo (mesas de diálogo, consulta a expertos, tribunales, mediación, negociación, entre otros tipos de diálogos), lo que muestra este hecho colectivo tan humano es que se reemplaza el criterio de validez por el de legitimidad. El mismo principio implícito que pareciera ser promovió Naess, Crawshay-Williams, Perelman y Toulmin en sus apuestas aplicadas y sociales.

\section{Notas}

(1) Este trabajo forma parte de los resultados de la participación en la investigación titulada La trama de la argumentación: claves conceptuales e implicaciones filosóficas, patrocinado por el Ministerio de Ciencia e Innovación de España, y cuyo director fue el profesor Luis Vega Reñón de la Universidad Nacional a Distancia (UNED) de Madrid.

(2) Así por ejemplo, la conferencia de 1995 se tituló Argumentation and Education; la de 1997 Argumentation and Rhetoric; la de 1999 Argumentation at the Century's Turn; la de 2001 Argumentation and its Applications; un caso especial fue la del 2003 que celebró el 25 aniversario del primer coloquio en Lógica Informal; también la de 2005 fue especial pues fue dedicada a Stephen Toulmin titulándose sin más como The Uses of Argument; para el 2007 se convocó a los especialistas bajo el título de Dissensus and the search for common ground; en 2009 el título fue Argument Cultures; y recientemente (2011) los investigadores se reunieron bajo el título Cognition and Community.

(3) Reynoso desmenuza crudamente, y no pocas veces de forma ácida, las propuestas de científicas sociales de, por ejemplo, Latour y Morin, acusándolos no sólo de leer incorrectamente las teorías de otros, sino sobre todo de crear metáforas débiles e ingenuas para explicar los fenómenos sociales.

(4) Hay una vasta bibliografía en estudios metafóricos desde que Lakoff y Johnson rehabilitaran el tema desde lo que llamaron una epistemología experiencialista. El razonamiento analógico, desde la perspectiva 
de la psicología del razonamiento y los estudios del pensamiento crítico, con fuerte énfasis experimental, ha dejado meridianamente claro el papel de este modo de expresión del raciocinio.

\section{Bibliografía}

Berk, U. 1979. Konstruktive Argumentationtheorie. Suttgart: Frommann-Holzboog.

Blair, A. 2011. Groundwork in the theory of argumentation. New York: Springer.

Cantú, P. y Testa, I. 2006. Teorie dell'argomentazione. Milano: Bruno Mondadori.

Crawshay-Williams, R. 1947. The comforts of unreason. London: Routledge \& Kegan Paul.

Crawshay-Williams, R. 1957. Methods and criteria of reasoning. An inquiry into the structure of controversy. London: Routledge and Kegan Paul.

Eemeren, van F. y Grootendorst, R. 1996. Developments in argumentation theory. En: van Benthem, J; van Eemeren, F; Grootendorst, R. y F. Veltman. Logic and argumentation. Amsterdam: Royal Netherlands Academy of Arts and Sciences, pp. 9-26.

Eemeren, van. F; Garssen, B; Haften, van P. y Krabbe, E. 2012. Fundamental of argumentation theory. New Jersey: LEA.

Eemeren, van F; Grootendorst, R. y Kruiger, T. 1978. Argumentatietheorie. Utrecht: Het Spectrum.

Fumerton, R. 2006. Epistemology. London: Blackwell Publishing.

Hamblin, C. 1970. Fallacies. London: Methuen \& Co. Ltd.

Johnson, R. 2000. Manifest rationality. A pragmatic theory of argument. New Jersey: Lawrence Erlbaum Associates, Inc.

Johnstone, H. 1968. Theory of argumentation. En: R. Klibansky (Ed.) La philosophie contemporaine. Firenze: La Nuova Italia Editore, pp. 177-184.

Kopperschmidt, J. 1980. Argumenttaion. Stuttgart: Kohlammer.

Lumer, C. 2005. Introduction: A map. Informal Logic 25(3): 189-212.

Naess, A. 1966. Communication and argument. Elements of applied semantics. London: Allen and Unwin.

Öhlschläger, G. 1979. Linguistische Überlgungen zu einer Theorie der Argumentation. Tübingen: Niemeyer.

Perelman, Ch. y Olbrechts-Tyteca, L. 2000. The new rhetoric. A treatise on argumentation. Notre Dame: University of Notre Dame Press.

Rehg, W. 2009. Cogent science in context. The science wars, argumentation theory and Habermas. Cambridge, Mass.: The MIT Press.

Schecker, M. 1977. Theorie der Argumentation. Tübingen: Narr.

Toulmin, S. 1958. The uses of argument. Cambridge: Cambridge University Press.

Toulmin, S; Rieke, R. y Janik, A. 1979. An introduction to reasoning. New York: Macmillan.

Walton, D. 2007. Media argumentation. Dialectic, persuasion, and rhetoric. Cambridge: Cambridge University Press.

Walton, D; Reed, C. y Macagno, F. 2008. Argumentation schemes. New York: Cambridge University Press.

Recibido el 28 de octubre de 2011

Aceptado el 7 de enero de 2012 